\title{
Sleep Disturbances in Women With Metastatic Breast Cancer
}

\author{
Cheryl Koopman, PhD, ${ }^{*}$ Bita Nouriani, MA, ${ }^{*}$ Vanessa Erickson, BA, ${ }^{\dagger}$ \\ Renu Anupindi, MD, ${ }^{\ddagger}$ Lisa D. Butler, PhD, ${ }^{*}$ Michael H. Bachmann, MD, DSc, ${ }^{*}$ \\ Sandra E. Sephton, PhD, ${ }^{\S}$ and David Spiegel, MD* \\ *Stanford University School of Medicine, Stanford, California, ${ }^{\dagger}$ University of Michigan Medical \\ School, Ann Arbor, Michigan, ${ }^{\ddagger}$ Austin State Hospital, Austin, Texas, and ${ }^{\S}$ University of Louisville \\ School of Medicine, Louisville, Kentucky
}

\begin{abstract}
We examined sleeping problems in women with metastatic breast cancer in relation to depression, social support, and salivary cortisol. Ninety-seven women with metastatic breast cancer were drawn from a larger study on the effects of group therapy on quality of life and survival. This study is based on the baseline assessments conducted prior to randomization into treatment conditions. Sleep, depression symptoms, and social support were assessed by self-reporting. Cortisol was assessed from saliva samples taken over a 3-day period. Medical status and demographic characteristics were also examined in relation to each sleep variable in multiple regression analysis. Most women (63\%) reported one or more types of sleep disturbance and $37 \%$ reported using sleeping pills in the previous 30 days. Problems with falling to sleep were significantly related to greater pain and depressive symptoms. Problems of waking during the night were significantly associated with greater depression and less education. Problems in waking/getting up were significantly associated with greater depressive symptoms and less social support. Sleepiness during the day was not significantly related to the variables in the regression model. Fewer hours of sleep were significantly associated with metastases to the bone, higher depressive symptoms, and more social support. Women who reported sleeping 9 or more hours per night, compared to those who reported a moderate amount of sleep (6.5-8.5 hours), had significantly lower 9 P.M. cortisol levels. Use of sleeping pills was more frequent among women reporting greater pain and
\end{abstract}

Address correspondence and reprint requests to: Cheryl Koopman, PhD, Department of Psychiatry and Behavioral Sciences, Stanford University School of Medicine, 401 Quarry Rd., MC: 5718, Stanford, CA 94305-5718, USA.

(C) 2002 Blackwell Publishing, Inc., 1075-122X/02/\$15.00/0

The Breast Journal, Volume 8, Number 6, 2002 362-370 depressive symptoms. These results suggest that women with metastatic breast cancer who are at higher risk for having sleeping problems are those who are less educated, in pain, depressed, have bony metastases, or lack social support.

Key Words: breast cancer, cortisol, depression, metastases, sleep, social support

$\mathrm{S}$ leep problems are persistent among long-term survivors of breast cancer, and such problems are associated with a poorer quality of life in this population (1). In addition to promoting better quality of life, undisturbed sleep appears essential for coping with cancer and symptoms such as pain, because disturbed sleep results in fatigue and lowers the threshold for experiencing negative stimuli and feelings (2). Broeckel et al. (3) found that, compared to women with no history of cancer, breast cancer patients who had formerly received chemotherapy reported more severe fatigue, and severe fatigue was significantly correlated with poorer sleep quality and it resulted in a poorer quality of life.

We have not found any published studies that have utilized either physiological or self-report measures to investigate the magnitude and severity of sleep disturbance specific to patients with metastatic breast cancer. The research participants in most published studies are patients with primary breast cancer as well as other types of malignancies, such as lung, colon, and prostate cancer. One such study (2) investigated 
sleep alterations among patients with any stage of breast or colon cancer. Of the 150 patients interviewed, $40 \%$ reported sleep problems during the previous month. Half of the patients interviewed reported sleep disturbances. Of those with sleep problems, $45 \%$ reported problems every night or almost every night, and $90 \%$ reported that the most common type of sleep problem was awakening during the night. Other common problems included sleeping fewer hours than normal $(85 \%)$ and difficulty falling back to sleep $(75 \%)$. In a similar study by Malone et al. (4), three-fourths of cancer patients surveyed experienced disturbances in sleep and rest.

The importance of sleep to both clinical and nonclinical populations has also been shown by studies examining physiological alterations associated with sleep disturbances. One significant physiological marker found to be correlated with sleep disturbance is an elevation of cortisol, a stress hormone associated with the hypothalamic-pituitary-adrenal (HPA) axis. Normally there is marked circadian variation in cortisol, with morning levels four to five times higher than those found in late evening $(5,6)$. Sleep disruption is associated with altered circadian rhythms of cortisol. For example, Leproult et al. (7) examined the effects of sleep loss on the HPA axis and found that acute partial or total sleep deprivation resulted in elevated cortisol levels the following evening. Specifically the findings show that sleep loss delays the recovery of the HPA axis from early morning stimulation. A similar study by Vgontzas et al. (8) examined the relationship between chronic insomnia and the activity of the stress response system and found that 24-hour urinary free cortisol levels were positively correlated with the degree of objective sleep disturbance. The physiological data collected from both of these studies indicate that sleep disturbance has a significant effect on the stress system and could potentially affect the resiliency of the stress response in individuals suffering from such disturbances.

Elevated cortisol levels (9) and loss of normal diurnal variation in cortisol (10) have been found among breast cancer patients. This flattening of cortisol profiles has been found to predict earlier mortality with metastatic breast cancer (11). Although in that sample the loss of diurnal variation was also associated with more selfreported awakenings during the night, we have been unable to find any other studies linking these altered cortisol levels with sleep disturbances in cancer patients. However, previous research suggests other factors to be associated with quality of sleep among cancer patients, including intensity of pain, feelings of anxiety and depression, and life stress $(12,13)$.

In an analysis of electroencephalogram (EEG) sleep profiles, Dew et al. (14) showed that individuals who were in an early stage of depression were significantly more likely to experience impaired sleep continuity and sleep architecture, while those who had experienced depression for a longer duration had less evident sleep disturbances that were still greater than found among nondepressed individuals. Furthermore, those with the poorest sleep were older and less educated, carrying a greater medical burden, and enjoying less social support, suggesting that demographic, medical, and psychosocial factors may also be associated with sleep disturbances.

Pain among patients with metastatic cancer has been found to be associated with fatigue (15), thus suggesting that disturbed sleep among this population may also be common. Portenoy et al. (16) found that $33 \%$ of their lung and colon cancer patients claimed persistent or frequent cancer-related pain during the previous 2 weeks, and more than half of the patients reporting pain also reported moderate or greater interference in sleep, mood, and enjoyment of life. The relationship of pain to sleep disturbances is supported by research with chronic pain patients finding that sleeping problems began after the onset of pain complaints (17).

In the present study, we present the self-reported patterns of sleep disturbance and associated depression, social support, and cortisol among women with metastatic breast cancer who volunteered to participate in a randomized clinical trial of group psychotherapy. Based on the general literature as well as the literature focusing on cancer patients, we hypothesized that among women with metastatic breast cancer, those reporting the most sleep disturbances would have greater depression and less social support, as well as higher salivary cortisol levels, compared to women experiencing less sleep disturbance. We examined cortisol levels early in the day (8 A.M.) and late in the evening (9 P.M.) because those times were proximal to the period during which most of the women would be sleeping. We also explored the relationships of pain, education, and medical status to sleep disturbances.

\section{MATERIALS AND METHODS}

\section{Participants}

Study participants were 97 women (mean age 53.0 years, $S D=11.0$, range 33-80 years) with confirmed metastatic or locally recurrent breast cancer drawn from 
a larger sample of 125 women participating in a randomized clinical trial examining the effects of group psychotherapy on psychosocial adjustment and survival. The analyses included all 97 women for whom we have all relevant data. The cortisol data for 21 women could not be included in the analysis either because they were currently taking drugs that are known to affect cortisol level, for example, steroids, or because we were unable to reliably assess the cortisol from their saliva. The data for an additional seven women were not used in this analysis because of missing data on one or more of the key measures. We compared the 97 women whose data were included in this study to those 28 women whose data were not included to determine whether they significantly differed on any of the other medical or demographic variables. We used $t$-tests to examine continuous variables (e.g., age and chi-square tests to examine categorical variables (e.g., marital status) and applied the Bonferroni correction for multiple comparisons. No significant differences in medical or demographic variables were found between women included in this study and women who were not included in this study.

Ethnic backgrounds of the women in this study included 90\% Caucasian, 6\% Asian American, 1\% African American, $1 \%$ Native American, and 2\% other. Fifty-three percent of participants were married, 35\% were separated, divorced, or widowed, $11 \%$ had never been married, and one participant classified herself as "other." The mean education level in this sample was 16.25 years $(\mathrm{SD}=2.6$, range $12-26$ years $)$, and mean annual household income was approximately $\$ 60,000$. For details on participant recruitment and inclusion/ exclusion criteria, please see Classen et al. (18). All participants provided informed consent in accordance with procedures approved by our institution's human subjects review panel.

\section{Procedure}

Data in the present report were collected at a baseline assessment prior to randomization into study conditions. Psychosocial assessments were conducted by self-report questionnaires. Cortisol assessments were performed on saliva samples collected by the participants at home over a 3-day period.

\section{Measures}

Demographic and Medical Variables. Demographic information, including current age, marital status, and education, was collected from all patients. Medical variables were collected and verified through chart review. For the purposes of the present report, we examined the number of months since diagnosis of recurrence/metastasis, the disease-free interval (in months), whether or not the site of metastasis was to bone (yes or no), whether the patient had received chemotherapy within the previous 2 months (yes or no), and degree of current pain. Degree of current pain ("experience of pain at this moment") was assessed with an item from the nine-item Pain Questionnaire (19), which used a 10-point visual analog scale that ranges from 1 (not noticeable) to 10 (excruciating-worst ever) to assess the degree of self-reported pain.

Sleep. The 27-item Sleep Questionnaire, which is based on the Structured Insomnia Interview developed by the Stanford University Medical School Sleep Disorders Clinic, was used to assess the quantity and quality of sleep. For the present report, data from five questions were used to assess the types of sleeping problems: "Do you have a problem getting to sleep at night?" "Do you have a problem because you wake up during the night?" "Do you have a problem with waking up and getting up in the morning?" "Do you have a problem with sleepiness during the daytime (feeling sleepy, struggling to stay awake in the daytime)?" How many hours of sleep do you usually get at night? The first four questions were answered on a 5 -point scale (1, none; 2 , slight; 3 , moderate; 4 , great; 5 , severe); the final question asked the participant to answer in hours and minutes. In addition to these measures we also assessed women's use of sleeping pills using an item on the Sleep Questionnaire that asked: During the last 30 days, how many times have you used some kind of sleeping pill in order to sleep better? Responses were 1, never; 2, less than two times a week; 3 , several times a week; 4, almost everyday; 5 , everyday.

Depression Symptoms. Depression symptoms were assessed with the 20-item Center for Epidemiological Studies Depression Scale (CES-D) (20). Participants were asked to indicate how they felt during the past week on items such as I felt sad. Response options were 0 , rarely/none of the time (less than 1 day); 1 , some or a little of the time ( 1 or 2 days); 2 , occasionally (3-4 days); 3 , most of the time (5-7 days). Research on the CES-D has supported its validity and reliability in assessing depression (20). We selected this scale because the items did not mimic the experience of physical illness (e.g., 
fatigue, weight loss). Internal consistency for the CES-D at baseline was found to be high (Cronbach's $\alpha=0.91$ ) in a subset of women participating in the larger study (unpublished data).

Social Support. Social support network size was assessed with the Single-Item Measure of Social Support (SIMSS), a measure that has been found to be an excellent predictor of mortality (21). The item reads, How many people do you have near that you can readily count on for real help in times of difficulty, such as watch over children or pets, give rides to hospital or store, or help if you are sick? Response options are 0,1 , $2-5,6-9$, or 10 or more. Responses of 0 or 1 indicate low levels of social support; $2-5$ or more indicate high levels.

Salivary Cortisol. The measurement of cortisol in saliva has been confirmed to reliably reflect free cortisol levels in blood (22). Patients collected saliva at home with salivette devices, consisting of a cotton swab fitted into a plastic holder resting inside a centrifuge tube. Samples were taken at $0800,1200,1700$, and 2100 hours on three consecutive days. Because sleep disturbance is most likely to affect cortisol secretion levels measured soon after awakening, and because sleep disturbance is most likely to be exaggerated by higher cortisol levels just before bedtime, only the 0800 ( 8 A.M.) and 2100 (9 P.M.) samples were used in the current analysis. Medications and stressors during the sampling period were assessed by questionnaire. Samples were centrifuged and aliquots frozen at $-70^{\circ} \mathrm{C}$. Cortisol levels were assessed by ${ }^{125} \mathrm{I}$ radioimmunoassay (11). Intra-assay coefficients of variation on three different saliva pools averaged $5.3 \%$, and the interassay coefficient was $12 \%$. Assay sensitivity was $0.008 \mu \mathrm{g} / \mathrm{dl}$.

\section{Data Analysis}

Hierarchical multiple regression analysis was used to analyze the sleep variables, which served as the dependent variables in these analyses. Four kinds of sleeping problems were examined: problems in falling asleep, problems with awakening in the night, problems waking/getting up in the morning, and sleepiness during the day, as well as the typical number of hours of sleep per day. No pair of these sleep variables was strongly correlated, although there were moderate correlations between several of the pairs. Having difficulty in getting to sleep was correlated with problems waking during the night $(r=0.39, p<0.001)$, with problems waking/ getting up in the morning $(r=0.23, p<0.05)$, and with the typical number of hours of sleep $(r=-0.36$, $p<0.001)$. Problems with waking up during the night were also related to problems waking/getting up in the morning $(r=0.22, p<0.05)$ and with the typical number of hours of sleep $(r=-0.33, p<0.001)$. Problems waking/getting up in the morning were also related to sleepiness during the day $(r=0.34, p<0.001)$. The magnitudes of these correlations demonstrated sufficient independence to allow us to conduct a multiple regression analysis on each of the sleep measures as an independent variable. We also examined as an alternative indicator of sleep problems the frequency of use of sleeping pills in the previous 30 days.

Independent variables were entered in four blocks. In the first block, we controlled for demographic characteristics (i.e., age, marital status, and education) and medical status (i.e., number of months since diagnosed, disease-free interval, site of recurrence being in the bone, having received chemotherapy within the previous 2 months, and current pain) by introducing these variables in a stepwise forward procedure. The second block used the simultaneous entry procedure to test the relationship of CES-D depression total scores and the SIMSS rating to each sleep variable. The third block offered the log transformed mean cortisol at 8 A.M. using the stepwise forward procedure. The fourth block used this same procedure to offer the log transformed mean cortisol at 9 P.M. We offered these cortisol values in separate blocks because they were significantly correlated with each other $(r=0.72, p<0.001)$ and we wanted to determine whether either cortisol value was related to women's sleep problems.

\section{RESULTS}

\section{Descriptive Statistics}

The descriptive statistics (and percentages where appropriate) for the independent and dependent variables are presented in Table 1 . The percentage of the women who reported each of the sleeping problems, based on reports that each of the problems was in the moderate to severe range, are as follows: $24.7 \%$ reported problems in falling asleep at night, $44.3 \%$ reported problems with waking in the night, $29.9 \%$ reported problems with waking and getting up in the morning, and $20.6 \%$ reported sleepiness during the day. Combining the women who reported any of these problems, $63 \%$ reported one or more sleep disturbance. Furthermore, 
Table 1. Medical, Psychosocial, and Sleep Characteristics of the Sample

\begin{tabular}{|c|c|c|c|}
\hline Variable & Percentage & Mean (SD) & Range \\
\hline $\begin{array}{l}\text { Time since diagnosis of } \\
\text { recurrence/metastases (months) }\end{array}$ & & $19.72(33.08)$ & $1-244.7$ \\
\hline Disease-free interval (months) & & $46.75(35.64)$ & $0-162.3$ \\
\hline Recurrence to bone & 38.1 & & \\
\hline $\begin{array}{l}\text { Had chemotherapy in } \\
\text { previous } 2 \text { months }\end{array}$ & 30.9 & & \\
\hline Pain in this moment & & $1.97(1.48)$ & $1-7$ \\
\hline CES-D total score & & $12.58(10.17)$ & $0-41$ \\
\hline SIMSS & & $3.40(1.05)$ & $1-5$ \\
\hline Mean raw cortisol ( $\mu \mathrm{g} / \mathrm{dl})$ & & & \\
\hline across time points & & $0.49(0.62)$ & $0.02-3.32$ \\
\hline 8 A.M. mean raw cortisol $(\mu \mathrm{g} / \mathrm{dl})$ & & $0.73(0.59)$ & $0.01-4.61$ \\
\hline 9 P.M. mean raw cortisol ( $\mu \mathrm{g} / \mathrm{dl})$ & & $0.34(0.75)$ & $0.02-5.58$ \\
\hline Problems in falling asleep & & $1.95(1.03)$ & $1-5$ \\
\hline $\begin{array}{l}\text { Problems with waking during } \\
\text { the night }\end{array}$ & & $2.37(1.03)$ & $1-5$ \\
\hline $\begin{array}{l}\text { Problems in waking up } \\
\text { in the morning }\end{array}$ & & $2.09(1.13)$ & $1-5$ \\
\hline $\begin{array}{l}\text { Problems with sleepiness } \\
\text { during the day }\end{array}$ & & $1.87(0.91)$ & $1-4$ \\
\hline Typical number of hours of sleep & & $7.48(1.22)$ & $4.5-10$ \\
\hline
\end{tabular}

$36.7 \%$ reported use of sleeping pills within the previous 30 days.

\section{Results of Multiple Regression Analyses}

The results of each multiple regression analysis is presented in Table 2, showing the final results after all blocks were offered to the analysis. Problems falling to sleep in the evening were significantly related to higher pain and CES-D total scores $(F[3,93]=6.86, p<0.001$, overall adjusted $\left.R^{2}=0.16\right)$. Problems waking during the night were significantly associated with greater CES-D scores and less education $(F[3,93]=6.29$, $p<0.001$, overall adjusted $\left.R^{2}=0.14\right)$. Problems waking/getting up in the morning were significantly associated with greater CES-D scores and less social support $(F[3,93]=12.01, p<0.001$, overall adjusted $R^{2}=0.26$ ). Sleepiness during the day was not significantly related to the variables in the regression model. Women who reported significantly fewer typical hours of sleep were significantly more likely to be those with bony metastases, with lower CES-D scores, and with less social support $(F[4,92]=4.23, p<0.01$, overall adjusted $R^{2}=0.12$ ). Frequency of use of sleeping pills in the previous 30 days was greater among women reporting depressive symptoms and pain $\left(F[3,93]=9.76, p<0.001\right.$, overall adjusted $\left.R^{2}=0.18\right)$. Salivary cortisol did not show any significant linear relationships with the sleep variables.

\section{Secondary Analysis of Typical Hours of Sleep}

We conducted a secondary analysis to examine the possibility that the typical number of hours slept might be nonlinearly related to the cortisol levels at 8 A.M. and 9 P.M. We categorized hours of sleep as low (less than 8 hours/night), moderate (6.5-8.5 hours/night), and high ( 9 or more hours per night). Then we used this recorded variable as an independent variable in one-way analysis of variance (ANOVA) to analyze its relationship to the cortisol levels at 8 A.M. and 9 P.M. Women who reported that they typically slept a high number of hours per night had lower mean log transformed 9 P.M. cortisol $(M=-1.22, \mathrm{SD}=1.12)$ compared to those who reported a moderate amount of sleep $(M=-2.10, \mathrm{SD}=0.90)$. This mean difference was statistically significant in the one-way ANOVA, where overall $(F[2,94]=4.35$, $p<0.05$, using the least square differences test (paired comparison mean difference $=0.88$, standard error $=$ $0.30, p<0.01)$. The mean $\log$ transformed 9 P.M. cortisol among those who reported that they typically slept a low number of hours $(M=-1.93, \mathrm{SD}=1.10)$ did not differ significantly from the other values. No significant differences were found for 8 a.m. cortisol by typical level of hours of sleep.

\section{DISCUSSION}

Sleep disturbances are important and often overlooked problems that constitute an aspect of quality of life for metastatic breast cancer patients. We found that these patients vary in their sleeping problems. Considerable numbers of the women reported each of the types of sleep disturbance examined in this study, ranging from $21 \%$ who reported experiencing sleepiness during the day to $44 \%$ who reported problems with waking during the night. Altogether, nearly two-thirds $(63 \%)$ of the participants in our study reported at least one of the sleep problems we assessed. As these percentages indicate, sleep disturbances are a common problem among women with metastatic breast cancer. Given this scale of the problem with sleep among these women, our findings regarding other factors related to sleep disturbance seem of particular interest for identifying women at high risk for sleep disturbance.

Difficulty falling asleep (early insomnia), reported by $25 \%$ of the women, was found to be significantly greater among those who reported greater pain. Furthermore, the women reporting pain also tended to use sleeping pills more frequently, another sign of sleep difficulty. A possible interpretation of these findings is 


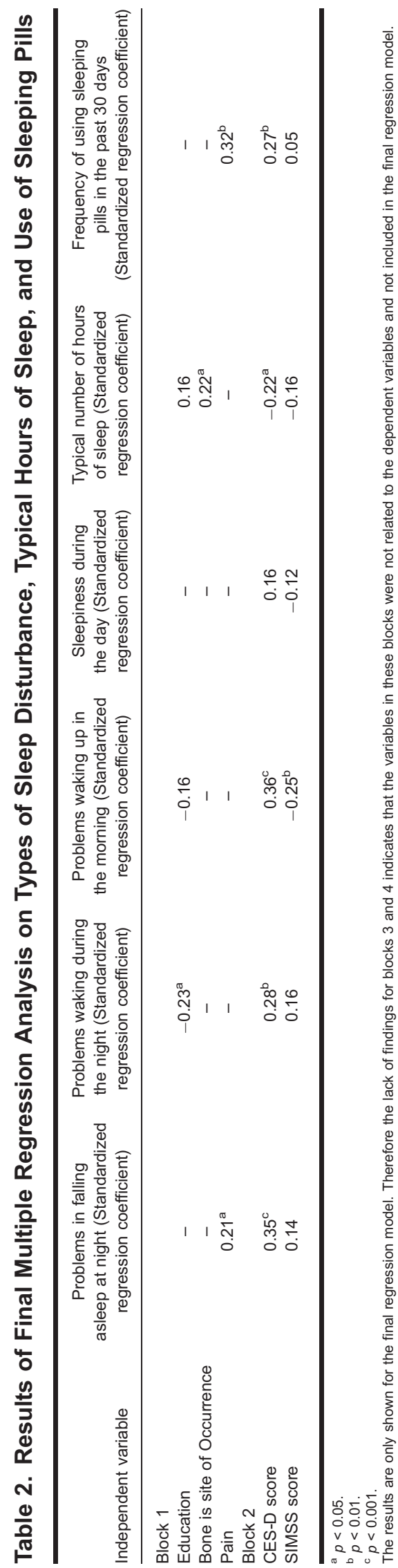

that pain may have been interfering with women being able to fall or remain asleep. This raises questions about the adequacy of pain management among these women, a concern that has been raised regarding the treatment of cancer patients in general. Inadequate pain management and its undertreatment in cancer patients remains a striking problem according to a recent report by the National Cancer Policy Board, an expert committee created by the Institute of Medicine and National Research Council (23), despite impressive scientific progress in knowledge about pain. According to a recent study, $36 \%$ of cancer patients with metastatic disease who had pain experienced severe enough pain to compromise their daily functioning (24). Thus sleep disturbances related to pain may benefit from adequate analgesia (25).

Another finding that raised questions about the adequacy of analgesia was that the typical number of hours that women slept was lower among those whose site of recurrence was in the bone. Bone metastases can be a further source of pain. Therefore the finding that fewer hours of sleep is related to having bone as the site of recurrence is consistent with the possibility that women may need more analgesia to alleviate pain that can interfere with sleep.

Cancer patients vary widely in their distress depending upon the organ affected and the stage of the disease. For pain, adequate analgesia should be provided according to the National Comprehensive Cancer Network guidelines, which are based on objective pain assessment and utilize both pharmacologic and nonpharmacologic interventions (26).

It was interesting that waking during the night (middle insomnia) was related to having less education. One explanation for this could be that a less educated woman may have a poorer understanding of the disease. Therefore its prognosis and available treatment modalities could increase her worries and fears, thereby exacerbating her sleep problems. An alternative explanation could be the association of lower income with less education, which in itself could reduce the availability of material resources for buffering stress which can interfere with sleep. To the extent that knowledge is helpful, providing education about breast cancer may assist women in adapting to the physiological and psychological sequelae of breast cancer. The potential value of such education is suggested by a finding in previous research that about three-fourths of women in a study group showed a positive change in attitude toward 
breast cancer after receiving group support and education for breast cancer (27).

In the present sample, the most striking relationships found were those of depressive symptoms being associated with greater sleep disturbance. Higher depression scores on the CES-D were associated with problems in falling asleep, waking during the night, difficulties in waking/getting up in the morning, less sleep, and more frequent use of sleeping pills. Using 16 as a cutoff score on the CES-D, which has been used to identify individuals with clinically significant symptoms of depression (20), we found that nearly one-third $(30 \%)$ of the women from our study sample met or exceeded this score. The origin of depressive symptoms in these patients could be multifactorial; these women suffer from advanced breast carcinoma and pain, as well as a variety of psychosocial factors. A number of previous studies have found evidence linking depression and pain $(15,28,29)$. Furthermore, it is thought that insomnia is caused by dysfunction of serotoninergic systems, a prominent component of the neurobiology of depression (30). Therefore it can be assumed that the changes in sleep architecture reported by our study sample may have been to some extent a consequence of depressive disorders. However, because the women in this sample were screened to exclude patients with current psychiatric morbidity, they may have less depressive symptoms than do metastatic breast cancer patients in general.

As hypothesized, sleep disturbance among women with metastatic breast cancer was associated with having less social support. The chief complaint of this subset of the sample was waking/getting up in the morning. Social support among these patients has been identified as a fundamental coping resource, where emotional support from a husband, family, and close friends is of utmost importance (31). This finding further underscores the need to evaluate the patient's support system. Apart from friends and family, health care professionals can also be an important source of informational and emotional support (31).

Nearly one-fourth of the women in our sample reported experiencing moderate to severe sleepiness during the daytime, but it was unrelated to other variables examined in the multiple regression analysis. This relatively high prevalence of daytime sleepiness is interesting. Perhaps a contributing factor was fluctuations in the levels of sedating medications the women were taking which we are unable to assess. This possibility led us to conduct a secondary analysis to compute correlation coefficients examining for relationships of sleep problems with a separate assessment of use of any sedating medications that had been reported within a 2-week window of completing the sleep measures. None of these relationships was statistically significant, but given the crudeness of our methods in this secondary analysis, the possibility still remains that fluctuations in the levels of sedating medications may contribute to their experiencing daytime sleepiness. Given the substantial percentage of women who reported daytime sleepiness, it is important that future research try to identify factors associated with this problem so that it can be better understood.

According to a large body of research, inadequate sleep leads to frustration, short temper, and a generally poor mood (32). Thus sleep disturbance may be accompanied by escalating cortisol levels that usually accompany distress. This is further suggested by findings that sleep deprivation is associated with a greater increase in cortisol the next day (33). One possible interpretation is that individuals who are experiencing sleep disturbance worry about this, further driving up their cortisol level. Another possible interpretation is that inadequate sleep directly drives up the cortisol level. Although this study was not designed to allow us to distinguish between these possible interpretations, a first step was to determine whether we could detect a significant difference in mean cortisol associated with sleep disturbances. We did not find in the multiple regression analyses that any of the sleep disturbance variables or typical hours of sleep was significantly related to early morning ( 8 A.M.) or late evening (9 P.M.) salivary cortisol among these women. However, a nonlinear relationship was suggested by the significantly lower 9 P.M. salivary cortisol found among the patients who reported typically sleeping 9 or more hours compared to women who reported typically sleeping 6.5-8.5 hours. These data support the findings of others showing that elevated evening cortisol levels are associated with sleep loss (7). As a next step in providing a better understanding of this relationship, future research should examine women's cognition, subjective distress, and changes in cortisol level in relation to sleep disturbances.

Methodologic limitations constrain our interpretation of this study's results. We screened out the patients with current severe depression, so findings from our sample are not necessarily representative of the nature or prevalence of mood disturbance in metastatic breast cancer patients. Also, our sample was not very diverse, 
either socioeconomically or ethnically. Therefore further research is required to examine the relationships of these sleep disturbance variables in more diverse populations of women with breast cancer. Because there was no objective measure evaluating sleep in this study, future research should also reexamine the relationships of the demographic, medical, and psychosocial variables examined in this study with more objective measures of sleep.

Side effects of cancer treatment, such as fatigue, which were not assessed in this study, have been identified as likely to be associated with sleep disturbances (25). Research is needed to provide a more comprehensive examination of factors associated with sleep disturbances. Our cross-sectional study design did not allow us to examine factors associated with improvement in sleep over time. Future longitudinal research focusing on women with metastatic breast cancer should examine the relationships between changes in psychosocial and neuroendocrine variables and changes in sleep disturbance. Given the prevalence of sleep problems among the women with metastatic breast cancer in this study, it is imperative that we determine the most effective ways to manage sleep problems in this population.

\section{Acknowledgments}

This study was funded by grant MH47226 from the National Institute of Mental Health (NIMH) and the National Cancer Institute (NCI), the John D. and Catherine T. MacArthur Foundation, and the Fetzer Institute. We also wish to express our appreciation to Elaine Miller as project director, Sue Dimiceli as data analyst, and Jane Benson, Hiroko Kameda, Krista Yocam, Lynne Lopresto, Leslie Smithline, Julie Seplaki, Karen Calde, and Greg Schaal as research assistants.

\section{REFERENCES}

1. Dow KH, Ferrell BR, Leigh S, Ly J, Gulasekaram P. An evaluation of the quality of life among long-term survivors of breast cancer. Breast Cancer Res Treat 1996;39:261-73.

2. Engstrom CA, Strohl RA, Rose L, Lewandowski L, Stefanek ME. Sleep alterations in cancer patients. Cancer Nurs 1999;22:143-48.

3. Broeckel J, Jacobsen PB, Horton J, Balducci L, Lyman G. Characteristics and correlates of fatigue after adjuvant chemotherapy for breast cancer. J Clin Oncol 1998; 16:1689-96.

4. Malone M, Harris AL, Luscombe DK. Assessment of the impact of cancer on work, recreation, home management and sleep using a general health status measure. J R Soc Med 1994;87:386-89.

5. Deuschle M, Schweiger U, Weber B, et al. Diurnal activity and pulsatility of the hypothalamus-pituitary-adrenal system in male depressed patients and healthy controls. J Clin Endocrinol Metab 1997;82:234-38.

6. Gritsko N, Shulga V, Ivanova L. Diurnal rhythms of the neuroendocrine system in professional riveters with different constitutional types. Cent Eur J Public Health 1995;3(suppl):132-34.

7. Leproult R, Copinschi G, Buxton O, Van Cauter E. Sleep loss results in an elevation of cortisol levels the next evening. Sleep 1997;20:865-70.

8. Vgontzas AN, Tsigos C, Bixler E, et al. Chronic insomnia and activity of the stress system: a preliminary study. J Psychosom Res 1988;45:21-31.

9. Van der Pompe G, Antoni MH, Heijnen CJ. Elevated basal cortisol levels and attenuated ACTH and cortisol responses to a behavioral challenge in women with metastatic breast cancer. Psychoneuroendocrinology 1996; 21:361-74.

10. Touitou Y, Bogdan A, Levi F, Benavides M, Auzeby A. Disruption of the circadian patterns of serum cortisol in breast and ovarian cancer patients: relationships with tumour marker antigens. Br J Cancer 1996;74:1248-52.

11. Sephton SE, Sapolsky RM, Kraemer HC, Spiegel D. Diurnal cortisol rhythm as a predictor of breast cancer survival. J Natl Cancer Inst 2000;92:994-1000.

12. Drewes AM, Nielsen KKDK, Arendt-Nielsen L, BirketSmith L, Hansen LM. The effect of cutaneous and deep pain on the electroencephalogram during sleep - an experimental study. Sleep 1997;20:632-40.

13. Monroe SM, Thase ME, Simons AD. Social factors and the psychobiology of depression: relations between life stress and rapid eye movement sleep latency. I Abnorm Psychol 1992;101:528-37.

14. Dew MA, Reynolds CF, Buysse DJ, et al. Electroencephalographic sleep profiles during depression. Effects of episode duration and other clinical and psychosocial factors in older adults. Arch Gen Psychiatry 1996;53:148-56.

15. Spiegel D, Sands S, Koopman C. Pain and depression in patients with cancer. Cancer 1994;1:2570-78.

16. Portenoy RK, Miransky J, Thaler HT, et al. Pain in ambulatory patients with lung or colon cancer. Cancer 1992;70:1616-24.

17. Morin CM, Gibson DG, Wade J. Self-reported sleep and mood disturbance in chronic pain patients. Clin J Pain 1998;14:311-14.

18. Classen C, Butler LD, Koopman C, Miller E, DiMiceli S, Giese-Davis J. Supportive-expressive group therapy and distress in patients with metastatic breast cancer patients: a randomized clinical intervention trial. Arch Gen Psychiatry 2001;58:494-501. 
19. Spiegel D, Bloom JR. Pain in metastatic breast cancer. Cancer 1983;52:341-45.

20. Radloff LS. The CES-D scale: a self-report depression scale for research in the general population. Appl Psychol Meas 1977;1:385-401.

21. Blake RL, McKay DA. A single item measure of social support as a predictor of morbidity. J Fam Prac 1986;22:82-84.

22. Kirschbaum C, Hellhammer DH. Salivary cortisol in psychoneuroendocrine research. recent developments and applications. Psychoneuroendocrinology 1994;19:313-33.

23. Chronicle News Services. Cancer care often skimps on symptoms. San Francisco Chronicle 2001; June 20:A4.

24. Cleary JF. Cancer pain management. Cancer Control 2000;7:120-31.

25. Hu D, Silberfarb PM. Management of sleep problems in cancer patients. Oncology 1991;5:23-28.

26. Benedetti C, Brock C, Cleeland C, et al. NCCN practice guidelines for cancer pain. Oncology 2000;14:135-50.
27. Samarel N, Fawcett J, Krippendorf K, et al. Women's perceptions of group support and adaptation to breast cancer. J Adv Nurs 1998;28:1259-68.

28. Chen ML, Chang HK, Yeh CH. Anxiety and depression in Taiwanese cancer patients with and without pain. $J A d v$ Nurs 2000;32:944-51.

29. Spiegel D. Cancer and depression. $\mathrm{Br} J$ Psychiatry 1996;168:109-16.

30. Thase ME. Treatment issues related to sleep and depression. J Clin Psychiatry 2000;11(suppl):46-50.

31. Fridfinnsdottir EB. Icelandic women's identifications of stressors and social support during the diagnostic phase of breast cancer. J Adv Nurs 1997;25:526-31.

32. Dement WC, Vaughan C. The Promise of Sleep. New York: Delacorte Press.

33. Goh VH, Tong TY, Lim CL, Low EC, Lee LK. Effects of one night of sleep deprivation on hormone profiles and performance efficacy. Mil Med 2001;166:427-31. 